* Ensaio

\title{
Sistema de Avaliação para a Qualificação do Sistema Único de Saúde (SUS)
}

\section{Afonso Teixeira dos Reis}

Coordenador-geral de Monitoramento e Avaliação (CGMA), do Departamento de Monitoramento e Avaliação do SUS (DEMAS), da Secretaria Executiva do Ministério da Saúde do Brasil. E-mail: afonso.reis@saude.gov.br.

\section{Paulo de Tarso Ribeiro de Oliveira}

Diretor do Departamento de Monitoramento e Avaliação do SUS (DEMAS), da Secretaria Executiva do Ministério da Saúde do Brasil. E-mail: paulo.oliveira@saude.gov.br.

\section{Paulo Eduardo Sellera}

Coordenador-geral de Gestão da Informação Estratégica (CGGIE), do Departamento de Monitoramento e Avaliação do SUS (DEMAS), da Secretaria Executiva do Ministério da Saúde do Brasil. E-mail: paulo.sellera@saude.gov.br.

DOI: $10.3395 /$ reciis.v6i2.Sup1.622pt

\section{Resumo:}

Ações e iniciativas de monitoramento e avaliação acompanham o desenvolvimento dos sistemas de saúde. O estágio atual de reconhecimento da complexidade da gestão de sistemas e serviços, em particular no caso brasileiro, devido à abrangência, ao volume de serviços e procedimentos e às características das disposições legais relativamente à organização, mostra a necessidade de uma política e de um sistema de monitoramento e avaliação. Em decorrência dessa necessidade, o Departamento de Monitoramento e Avaliação do SUS (DEMAS) da Secretaria Executiva do Ministério da Saúde, vem sistematizando e apresenta a proposta de um "Sistema de Avaliação para a Qualificação do SUS". O sistema de avaliação do SUS se constitui por um conjunto de programas de avaliação, relativamente independentes, mas relacionados, concatenados e complementares entre si, de modo a formarem um complexo que vise produzir, por meio de avaliações, um conjunto de informações necessárias e estratégicas ao desenvolvimento e qualificação do SUS, quanto ao cumprimento de seus princípios e diretrizes. A apresentação e análise descritiva apresentadas neste artigo incluem os seguintes componentes: o Índice de Desempenho do Sistema Único de Saúde - IDSUS; o Programa Nacional de Avaliação de Serviços de Saúde (PNASS); a Pesquisas nacionais de avaliação do acesso e de satisfação dos usuários; e o Programa Nacional de Melhoria do Acesso e da Qualidade da Atenção Básica (PMAQ). As iniciativas apresentadas pretendem sinergia entre si e a melhoria da capacidade de gestão no Sistema Único de Saúde (SUS).

Palavras-chave: monitoramento e avaliação em saúde, gestão do sistema único de saúde, gestão de ações e serviços de saúde, ministério da saúde do brasil. 


\section{Introdução}

Os saberes e ações de monitoramento e avaliação há muito estão presentes na área da saúde, assim como na história do sistema de saúde brasileiro. Diferentes concepções têm em comum que avaliar consiste em verificar, medir, determinar o valor de algo; a partir de um parâmetro (preceito legal, ótimo, padrão de referência, objetivo, etc.). Pode-se afirmar que o núcleo de qualquer avaliação, consiste na comparação do avaliado com um parâmetro definido, podendo ser com o próprio avaliado no tempo ou com seus semelhantes. Para essa comparação, em geral, utiliza-se de medidas, calculadas em determinado ponto de um período mais espaçado de tempo, expressas por indicadores quantitativos e, ou qualitativos.

Monitorar consiste em análises longitudinais que buscam produzir informações que revelem o curso ou desenvolvimento de algo no tempo, isto é, consiste em acompanhar o monitorado de forma mais frequente, por meio de observações, registros, medições e compilações.

Pode-se afirmar que monitoramento e avaliação são faces de um mesmo processo. Se monitorar é acompanhar o desenvolvimento de algo, isto significa compará-lo em sua evolução. Portanto, monitorar é também avaliar, pois um das formas de avaliar é comparar o desenvolvimento de algo no tempo. Por outro lado, acompanhar avaliações sucessivas, pode ser tomado como uma das formas de monitorar.

Embora muitas iniciativas e experiências estejam em curso, processos sistêmicos e periódicos de avaliação e monitoramento do Sistema Único de Saúde - SUS encontram-se pouco desenvolvidos.

Já é pratica frequente o uso de dados, indicadores, assim como análises e divulgação desses. No entanto, as informações geradas pouco orientam a tomada das decisões, assim como pouco se prestam para a qualificação dos serviços e ações da Vigilância e da Atenção à Saúde, e mais raro ainda, serem fontes para novos conhecimentos. Há, assim, no SUS a necessidade de produção das informações estratégicas para a gestão, despontando entre essas, os resultados de avaliações bem estruturadas, periódicas e contínuas, produtos de um sistema de avaliação.

Assumindo como um item imprescindível ao desenvolvimento da Política de Monitoramento e Avaliação do Sistema Único de Saúde, o Departamento de Monitoramento e Avaliação do SUS (DEMAS) da Secretaria Executiva do Ministério da Saúde, vem sistematizando e apresenta a proposta de um "Sistema de Avaliação para a Qualificação do SUS". Essa ação, de caráter estratégico e para apoio à gestão no SUS, decorre das competências do Departamento atribuídas pelo artigo 10, do Decreto 7.530/11, quais sejam:

I - coordenar a formulação e execução da Política de Monitoramento e Avaliação do SUS;

II - coordenar os processos de elaboração, negociação, implantação e implementação de normas, instrumentos e métodos necessários ao fortalecimento das práticas de monitoramento e avaliação do SUS;

III - articular e integrar as ações de monitoramento e avaliação executadas pelos órgãos e unidades do Ministério da Saúde; 
IV - desenvolver metodologias e apoiar iniciativas que qualifiquem o processo de monitoramento e avaliação do SUS;

V - viabilizar e coordenar a realização de estudos e pesquisas visando à produção do conhecimento no campo do monitoramento e avaliação do SUS;

VI - desenvolver ações com órgãos de controle interno e externo, com outros órgãos da Administração Federal e com entidades das áreas de informação e avaliação, visando a ampliar a qualidade do monitoramento e avaliação do SUS;

VII - participar da coordenação do processo colegiado de monitoramento, avaliação e gestão das informações do SUS; e

VIII - sistematizar e disseminar informações estratégicas para subsidiar a tomada de decisão na gestão federal do SUS.

A apresentação dessa proposta inclui a descrição de objetivos, da configuração da política e das principais iniciativas que estão sendo desenvolvidas, assim como a análise dos principais efeitos esperados com a sua efetiva implantação.

\section{Objetivos do Sistema de Avaliação para a Qualificação do SUS}

- Avaliar o SUS quanto ao cumprimento de seus princípios: universalidade do acesso, integralidade da atenção, igualdade e equidade e das diretrizes: regionalização, hierarquização, comando único por esfera de gestão e responsabilidade tripartite.

- Contribuir para a qualificação do SUS, no qual qualificar seja tomado em seus dois sentidos: o de encontrar a qualidade atual, assim como induzir melhorias, ou seja, de buscar acréscimos de graus de qualidade ao encontrado. Na medida em que se avalia, que se monitora, encontram-se problemas e deficiências, apontando para os gestores quais as prioridades de ação que imprimirão mais qualidade ao SUS;

- Subsidiar os gestores com informações que auxiliem o desenvolvimento de políticas, estratégias e programas necessários à efetivação dos princípios e diretrizes do SUS;

- Subsidiar os gestores na análise da situação de saúde e no planejamento da produção de serviços e ações de saúde, segundo necessidades e direitos dos cidadãos;

- Subsidiar a busca de maior eficiência e efetividade do SUS em produzir saúde para a população brasileira;

- Contribuir com as ações de Regulação, Controle, Avaliação e Auditoria do SUS;

- Contribuir com os órgãos de controle interno e externo da administração pública.

- Subsidiar, com informações estratégicas, o desenvolvimento das ações de participação e controle social. 


\section{Objetivos específicos}

- Avaliar o desempenho do SUS que atende os residentes dos municípios, regionais de saúde, estados, regiões brasileiras e no país;

- Avaliar o acesso e a efetividade nos diferentes níveis de atenção: básica, especializada ambulatorial e hospitalar e urgências e emergências;

- Avaliar os estabelecimentos de saúde, de todos os níveis da atenção, quanto à adequação de estrutura, processos e resultados;

- Avaliar o desempenho de equipes de saúde na produção do cuidado à saúde;

- Avaliar os graus de dificuldades de acesso e de satisfação dos usuários quanto à qualidade dos serviços e ações de saúde recebidos, em todos os níveis da atenção;

- Induzir, pelo uso sistemático, a melhoria da qualidade dos dados de gestão, financiamento, estrutura (estabelecimentos, redes, força de trabalho, etc.), da vigilância e atenção à saúde.

\section{Sistema de Avaliação para a Qualificação do SUS}

Um sistema de avaliação do SUS pode ser entendido como um conjunto de programas de avaliação, relativamente independentes, mas relacionados, concatenados e complementares entre si, de modo a formarem um complexo que vise produzir, por meio de avaliações, um conjunto de informações necessárias e estratégicas ao desenvolvimento e qualificação do SUS, quanto ao cumprimento de seus princípios e diretrizes.

O Sistema de Avaliação para a Qualificação do SUS teria como componentes:

- Índice de Desempenho do Sistema Único de Saúde - IDSUS

- Programa Nacional de Avaliação de Serviços de Saúde (PNASS)

- Pesquisas nacionais de avaliação do acesso e de satisfação dos usuários

- Programa Nacional de Melhoria do Acesso e da Qualidade da Atenção Básica (PMAQ)

\section{Índice de Desempenho do Sistema Único de Saúde - IDSUS}

O IDSUS é o programa que visa avaliar o desempenho do SUS quanto ao cumprimento de seus princípios: universalidade do acesso, integralidade da atenção, equidade e das diretrizes: regionalização, hierarquização, comando único por esfera de gestão e responsabilidade tripartite; a partir de indicadores calculados com dados dos Sistemas de Informações Nacionais ${ }^{1}$. Essa avaliação busca medir, aproximadamente, a distância entre o SUS existente e o SUS que atenderia a todos os brasileiros em todas suas necessidades de ações e serviços de saúde.

\footnotetext{
${ }^{1}$ Fonte de dados dos indicadores do IDSUS: Cadastro Nacional de Estabelecimentos de Saúde (CNES); Sistema de Informação Ambulatorial (SIA); Sistema de Informação de Agravos de Notificação (SINAN); Sistema de Informação do Programa Nacional de Imunização (SI-PNI); Sistema de Informação Hospitalar (SIH); Sistema de Informação sobre Mortalidade (SIM); Sistema de Informação sobre Nascido Vivo (SINASC); Instituto Brasileiro de Geografia e Estatística (IBGE); Ministério do Desenvolvimento Social e Combate a Fome (MDS)
} 
O núcleo elementar da avaliação realizada pelo IDSUS é o "SUS que atende os residentes de cada município brasileiro", pois contempla, além da Atenção Básica (toda realizada no município), a Atenção Especializada, Ambulatorial e Hospitalar, realizada tanto no próprio município, quanto a que é encaminhada para outros municípios, polos de uma região, de um estado ou nacional.

Em decorrência desse foco avaliativo, ressalta-se que o IDSUS não avalia apenas o sistema público de saúde existente nos territórios dos municípios, isoladamente, mas toda rede pública integrada e hierarquizada segundos os níveis de atenção, organizadas nas regiões de saúde e até para além dessas, isto é, avalia o conjunto de ações e serviços de saúde articulados em níveis de complexidade crescente, com a finalidade de garantir a integralidade da assistência à saúde, enquanto Rede de Atenção à Saúde, como definida, no Decreto no 7.508, de 28 de junho de 2011.

Assim, o IDSUS, tendo como a menor unidade avaliada o "SUS que atende os residentes de cada município brasileiro", (que inclui toda atenção regionalizada), avalia, também, o desempenho do SUS nos estados, regiões e no país, por meio da média, dos resultados da avaliação do "SUS que atende os residentes de cada município brasileiro", ponderada pelas respectivas populações.

Em síntese, o IDSUS tem como finalidades: (i) avaliar o desempenho do SUS nos municípios, regionais de saúde, estados, regiões e no país; (ii) avaliar o acesso e a efetividade nos diferentes níveis de atenção: básica, especializada ambulatorial e hospitalar e urgências e emergências; (iii) expressar essa avaliação por meio de indicadores simples e compostos; (iv) fazer avaliação para detectar as deficiências, visando melhorias e não apenas para classificar; (v) fazer uma avaliação atrelada a um pacto de compromissos, pois os indicadores do IDSUS terão metas definidas no Contrato Organizativo de Ação Pública - COAP, que visa organizar as ações e serviços de saúde especializados em redes regionalizadas e hierarquizadas, segundo o Decreto no 7.508.

A avaliação de desempenho pelo IDSUS, inspirada na dimensão de Avaliação de Desempenho do Sistema de Saúde do PRO-ADESS ${ }^{2}$, fixou como foco a avaliação do cuidado em saúde, por meio indicadores selecionados capazes de mensurar o acesso potencial ou obtido e a efetividade das ações e serviços de saúde, regionalizados e realizados nos níveis da Rede de Atenção à Saúde, para os residentes de cada município brasileiro.

O modelo avaliativo do IDSUS conta com um bloco de caracterização, segundo a homogeneidade dos municípios brasileiros, que foram agrupados em relação às semelhanças socioeconômicas, do perfil de mortalidade infantil e da estrutura do sistema de saúde existente no território municipal. Baseado nas outras três dimensões do PRO-ADSESS: Determinantes da Saúde, Condições de Saúde da População, Estrutura do Sistema de Saúde, esse bloco tem por objetivo fazer uma breve contextualização do desempenho encontrado no "SUS que atende os residentes de cada município brasileiro", portanto um recurso de análise dos resultados, não sendo utilizado para avaliar ou pontuar o desempenho do SUS.

Para a avaliação de desempenho do SUS, focada no cuidado em saúde, foram selecionados 24 indicadores (14 de acesso potencial ou obtido e 10 de efetividade), distribuídos entre a atenção básica, as atenções ambulatorial e hospitalar e a urgência e emergência.

Além dos vinte e quatro indicadores simples, o IDSUS conta com sete indicadores compostos, quais sejam:

\footnotetext{
${ }^{2}$ Projeto Desenvolvimento de Metodologia de Avaliação do Desempenho do Sistema de Saúde Brasileiro (PRO-ADESS), da Associação Brasileira de Pós Graduação em Saúde Coletiva (Abrasco), coordenado pelo Instituto de Comunicação e Informação Científica e Tecnológica em Saúde (ICICT), da Fundação Oswaldo Cruz (Fiocruz).
} 
- Índice Acesso potencial ou obtido na Atenção Básica

- Índice Acesso obtido na Atenção Ambulatorial e Hospitalar de Média Complexidade

- Índice Acesso obtido na Atenção Ambulatorial e Hospitalar de Alta Complexidade, Referência de Média e Alta Complexidade e Urgência e Emergência.

- Índice de Efetividade da Atenção Básica

- Índice de Efetividade da Atenção de Média e Alta Complexidade, Urgência e Emergência.

Esses cinco índices permitem o cálculo do Índice de Acesso potencial ou obtido do SUS, que avalia o acesso, em geral, e o cálculo do Índice de Efetividade do SUS, que avalia a efetividade da atenção, também, como média geral.

Os primeiros resultados do IDSUS foram publicados em primeiro de março de 2012 e referem-se aos dados do período de 2007 a 2010, portanto, indicam a situação do "SUS que atendeu os residentes de cada município brasileiro" até 2010. Esses resultados devem ser tomados como resultados aproximados de toda a história de construção do SUS e não apenas como resultado de uma ou da última gestão do SUS municipal, estadual e federal. Deve-se salientar também que eles não contemplaram as melhorias que podem ter ocorrido nos anos de 2011 e 2012 (os dados desses anos ainda não estavam completos no início de 2012).

Os pesos aos 24 indicadores que compõem o IDSUS foram atribuídos por meio da Análise de Componentes Principais, que se mostrou pertinente método que aponta as diferenças entre o "SUS que atende os residentes de cada município brasileiro", as diferenças de acesso e efetividade tanto entre os níveis de atenção quanto entre as regiões. Os indicadores compostos do IDSUS advindos da aplicação desse método, a construção dos mapas do IDSUS, a análise dos resultados por clusters de municípios permitem desvendar as iniquidades que devem ser superadas, isto é, possibilitam buscar maior equidade no SUS.

De forma geral, os primeiros resultados IDSUS apontaram que o maior problema do SUS, ainda é o de acesso, que a atenção básica, na maioria dos municípios brasileiros está melhor do que a atenção especializada. Assim, o IDSUS apontou que muito deve ser melhorado na regionalização, isto é, que se deve melhorar o acesso de residentes em cidades que não têm a atenção especializada, às cidades vizinhas, com mais infraestrutura de média e alta complexidade, ambulatorial e hospitalar, assim como melhorar o acesso dos residentes desses municípios polos de referência.

O IDSUS apontou os municípios, que além da atenção especializada, tem deficiências, também, na atenção básica, e que a deficiência da atenção especializada é maior na região Norte, Nordeste e Centro Oeste do que na Região Sudeste e Sul do Brasil.

O Ministério da Saúde pode decidir por alguma premiação por desempenho, a partir dos resultados do IDSUS, mas ela deverá se dar segundo o alcance de metas, isto é, da mudança do estágio encontrado pela avaliação até 2010 (ou subsequentes) para uma situação melhor. Portanto, não haverá penalização para um IDSUS baixo, mas os gestores receberiam incentivos proporcionais aos percentuais de alcance das metas. É importante ressaltar que não haverá metas nacionais, uniformes para todos, mas metas regionais, pactuadas nos Colegiados Regionais, por meio do COAP, considerando as dificuldades e especificidades do SUS nos municípios e regiões de saúde. 
Tais metas devem ser entendidas com passos para se atingir objetivos, expressos pelos parâmetros, estes sim, nacionais.

Enquanto indicador composto, expresso pelas notas, de 0 a 10, o IDSUS permite a todos, independente do grau de escolaridade, ter uma medida de como está atenção realizada pelo SUS, no município onde ele mora, incluindo quando ele precisar ser atendido em outro município. Esse conhecimento rápido, dado pela nota do "SUS que atende os residentes de cada município brasileiro" poder ser um poderoso instrumento de cobrança por melhorias, seja por qualquer cidadão, seja por aquele que está nos Conselhos de Saúde. Assim podemos dizer que as notas do IDSUS (que não é única, mas várias notas de acesso e efetividade da atenção básica e especializada) são fortes catalizadores de mobilização e de defesa do SUS, em prol de melhorias da atenção à saúde prestada por esse.

Essa característica de síntese, percepção e mobilização, também se fez presente no meio político: executivo, legislativo e gestores do SUS. Pode-se afirmar que desde sua criação, poucas vezes o SUS foi objeto de tanta preocupação por parte dos prefeitos, vereadores, deputados, governadores e mesmo no governo federal. Embora a preocupação com as eleições municipais, de 2012, a avaliação do IDSUS, ao apontar as dificuldades do SUS, põe em pauta a necessidade de ações para superá-las.

Mais do que um ranking, mais do que um conjunto de dados ou indicadores, mais do que um conjunto de fotografias, menos do que uma explicação exaustiva, a tentativa do IDSUS é de formular um conjunto de questionamentos sobre a realidade do "SUS que atende os residentes de cada município, região ou estado brasileiro". Como texto que oferece uma visão aproximada do SUS, espera-se que o IDSUS se consolide como uma das práticas de avaliação e monitoramento no sentido de identificar os principais problemas do SUS e, principalmente, reponha a necessidade de pactos entre os gestores, com metas e compromissos para a contínua construção do SUS que responda mais, aos direitos de cidadania e de saúde do povo brasileiro.

\section{Programa Nacional de Avaliação de Serviços de Saúde - PNASS}

A Secretaria de Atenção à Saúde do Ministério da Saúde, através do Departamento de Regulação, Avaliação e Controle de Sistemas - DRACS, a partir de 2004 reformulou o Programa Nacional de Avaliação de Serviços Hospitalares - PNASH, iniciado em 1998 e passou a denominá-lo Programa Nacional de Avaliação de Serviços de Saúde - PNASS.

O PNASS fixou como objetivo avaliar os serviços de saúde do Sistema Único de Saúde, buscando a apreensão mais completa e abrangente possível da realidade dos mesmos, buscando avaliar estruturas, processos e resultados relacionados ao risco, acesso e satisfação dos cidadãos diante dos serviços e estabelecimentos de saúde. Quatro dimensões foram avaliadas, sendo elas:

- Padrões de conformidade,

- Indicadores de produção,

- Pesquisa de satisfação dos usuários e

- Pesquisa das condições e relações de trabalho. 
O PNASS foi instituído pela portaria no 382/6M de 10/03/05, prevendo avaliar 9.747 serviços de saúde. Até junho de 2006, tinham sido respondidos 6.191 roteiros de padrões de conformidade pelos gestores municipais / estaduais.

A tabela 1 demonstra o percentual de respostas por tipo de avaliação.

Tabela 1 - Percentual de respostas do PNASS segundo o tipo de avaliação. Brasil, 2006

\begin{tabular}{|l|c|c|c|c|}
\hline & $\begin{array}{c}\text { Auto-Avaliação } \\
\%\end{array}$ & $\begin{array}{c}\text { Avaliação dos } \\
\text { Gestores \% }\end{array}$ & $\begin{array}{c}\text { Satisfação dos } \\
\text { usuários \% }\end{array}$ & $\begin{array}{c}\text { Relações de } \\
\text { Trabalho \% }\end{array}$ \\
\hline Não iniciada & 75,34 & 32,16 & 35,80 & 35,16 \\
\hline Em andamento & 1,5 & 4,3 & 6,3 & 6,3 \\
\hline Finalizada & 23,09 & 63,51 & 58,19 & 58,9 \\
\hline Total & 100,0 & 100,0 & 100,0 & 100,0 \\
\hline
\end{tabular}

Fonte: MS/SAS/DRAC/CGRA/SIPNASS - http://pnass.datasus.gov.br

O PNASS, embora não tenha tido uma continuidade, não deixa de ser uma importante iniciativa do Ministério da Saúde para avaliar os serviços de saúde e sua retomada é de fundamental importância, pois tem potência para fornecer uma rica base de dados, possibilitando a construção de indicadores de avaliação dos estabelecimentos de saúde, da satisfação dos usuários desses serviços, contribuindo, significativamente, para uma avaliação mais completa do desempenho dos sistemas de saúde. Pode-se apontar como causas do PNASS não ter tido pleno êxito:

1. Toda a coleta e digitação dos dados do PNASS, em software específico e online elaborado pelo Ministério da Saúde, ficaram sob responsabilidade dos gestores municipais e, ou estaduais. Estes não contavam com quantitativos adequados de trabalhadores para executar tal tarefa;

2. Pouca integração da proposta PNASS na rotina das atividades das Vigilâncias Sanitárias municipais e estaduais, em especial quanto à verificação do componente - Padrões de conformidade dos estabelecimentos;

3. Pouco desenvolvimento do componente - Indicadores de produção, com poucas testagens de indicadores da base SIA e SIH;

4. Não priorização e ou descontinuidade por parte do Ministério da Saúde no pleno desenvolvimento do programa, incluindo nula vinculação com qualquer política de atenção desenvolvida;

Desde 2010, o MS por meio da Fiocruz, contratou Consórcio Brasileiro de Acreditação - CBA para elaborar uma proposta de reestruturação do PNASS. Recentemente foi entregue tal proposta, após a testagem da metodologia em alguns hospitais distribuídos nas regiões brasileiras. Tal proposta, baseada na metodologia de acreditação de hospitais, embora pareça ser de difícil viabiliazação em sua plenitude, devido à necessidade de treinamento específico, pode trazer importantes subsídios à reformulação do PNASS, em especial quanto aos aspectos importantes e pertinentes à avaliação de qualidade dos serviços de saúde, baseada nos "Fundamentos Internacionais de Qualidade e Segurança no Cuidado ao Paciente"

Partindo das considerações acima, pode-se propor para a reestruturação do PNASS 
1. Seleção e testagem de um conjunto de indicadores tendo como base o SIA, o SIH e o SCNES. Testar a viabilidade de cálculo desses indicadores na base de dados da Comunicação da Internação Hospitalar, do SCIH;

2. Articular a reestruturação do PNASS com a proposta da ANS de avaliação de qualidade dos prestadores de serviços;

3. Utilizar toda a experiência e metodologias estatísticas do Índice de Desempenho do SUS IDSUS, incluindo o desenvolvimento de indicadores compostas e a pontuação a partir da comparação com parâmetros;

4. Reformular, em conjunto com a Vigilância Sanitária, o componente - Padrões de conformidade dos estabelecimentos, articulando a verificação desses às atividades de fiscalização e licenciamento dos estabelecimentos;

5. Incorporar na verificação, por parte das Vigilâncias Sanitárias, itens da proposta de qualificação do CBA;

6. Desenvolver módulos de verificação de padrões de conformidade necessários aos processos de habilitação e credenciamento de estabelecimentos para execução de ações e serviços especializados, em articulação com as áreas deda Atenção Especializada e de Regulação, Controle e Avaliação;

7. Renovar a metodologia de coleta e digitação dos dados do PNASS, usando computadores de mão, em rede online, como da experiência do IBGE para o censo 2010;

8. Desenvolver o módulo de pesquisa de satisfação dos usuários dos serviços por meio da experiência da Carta SUS e das pesquisas de satisfação das parturientes realizadas pela Ouvidoria do SUS

\section{Avaliação do acesso aos serviços e satisfação dos usuários}

Quanto à avaliação do acesso e satisfação aos serviços e ações de saúde no SUS, por meio de entrevista direta com os cidadãos brasileiros, conta-se com alguns dados periódicos (a cada 5 anos) da Pesquisa Nacional por Amostra de Domicílio - Suplemento Saúde (PNAD Saúde), do IBGE, que está sendo reformulada para constituir a Pesquisa Nacional de Saúde - PNS e, também, por algumas pesquisas amostrais, ocasionais, como a realizada pelo IPEA, em novembro de 2010.

Apesar da importância e significação dessas pesquisas, ainda se carece de uma pesquisa amostral que avalie aspectos do acesso aos serviços e a satisfação dos beneficiários, que além de periódica, fosse realizada em intervalos menores de tempo e fosse representativa, no mínimo do "SUS que atende os residentes de cada município brasileiro". A PNAD Saúde obtém resultados para grandes regiões, unidades da federação, regiões metropolitanas selecionadas e Distrito Federal.

Com objetivo de realizar inquéritos amostrais que avalie o acesso, utilização e satisfação do usuário do SUS, propõe-se:

- Realizar inquérito populacional, periódico (a cada 3 anos) amostral, enxuto em seu questionário e que avalie aspectos essenciais do acesso e satisfação dos usuários do "SUS que atende os residentes de cada município brasileiro" 
- Esse inquérito para um intervalo de confiança de 95\% e erro amostral de 5\% seria de 384 entrevistados, atendidos pelo SUS, por município brasileiro, que somaria 2.136 .960 entrevistados para todos os municípios brasileiros e que, se for pensado numa reposição de mais ou menos $50 \%$ (experiência Pesquisa amostral de acesso e satisfação dos usuários do SUS, realizada pela Ouvidoria do MS), pela não resposta ou por não ter siso atendido pelo SUS, daria em torno de 3,2 milhões de entrevistados;

- Esse tamanho da mostra permite obter resultados, estatisticamente significantes, referentes à atenção prestada, pelo SUS , a todos os entrevistados de um município, independente do nível da atenção. Para territórios maiores (regiões de saúde, estados, regiões e país), quando se somaria as respostas dos entrevistados de cada município, obter-se-ia resultados, estatisticamente significantes, para cada nível da atenção (básica, especializada ambulatorial e hospitalar, urgência e emergência)

- O modelo de questionário, baseado na Pesquisa amostral de acesso e satisfação dos usuários do SUS, realizada pela Ouvidoria do MS, no segundo semestre de 2011 e no modelo da Carta SUS seria único e discriminaria o tipo de estabelecimento de saúde buscado pelo usuário, nos últimos doze meses. Caso tenha usado mais de um, o usuário seria convidado a responder um questionário para cada tipo de estabelecimento (Anexo I, abaixo);

- A coleta dos dados seria feita por 4 vias, excludentes entre si para o mesmo entrevistado e somatórias entre si para compor o tamanho da amostra: mensagem SMS (celular), auto preenchimento pelo Disque saúde 136, entrevista online via telefone ou celular e entrevista presencial para localidades com baixa cobertura populacional de telefonia;

- Esse inquérito não eliminaria a Carta SUS e a pesquisa com parturiente SUS, que continuariam como canal de comunicação direta entre usuário e Ministério da Saúde e coletaria dados relacionados ao atendimento, discriminados por estabelecimento, que seria, a base da avaliação de satisfação do usuário do PNASS, além de permitirem avaliações específicas.

\section{Programa Nacional de Melhoria do Acesso e da Qualidade da Atenção Básica (PMAQ)}

O Programa Nacional de Melhoria do Acesso e da Qualidade da Atenção Básica é um Programa que procura induzir a instituição de processos que ampliem a capacidade das gestões federal, estaduais e municipais, além das Equipes de Atenção Básica, em ofertarem serviços que assegurem maior acesso e qualidade, de acordo com as necessidades concretas da população.

O Programa busca induzir a ampliação do acesso e a melhoria da qualidade da atenção básica, com garantia de um padrão de qualidade comparável nacional, regional e localmente de maneira a permitir maior transparência e efetividade das ações governamentais direcionadas à Atenção Básica em Saúde em todo o Brasil.

O PMAQ está organizado em quatro fases que se complementam e que conformam um ciclo continuo de melhoria do acesso e da qualidade da atenção primária $A B$ (Adesão e Contratualização; Desenvolvimento; Avaliação Externa; e Recontratualização).

A primeira fase do PMAQ consiste na etapa formal de adesão ao Programa, mediante a contratualização de compromissos e indicadores a serem firmados entre as Equipes de Atenção 
Básica com os gestores municipais, e destes com o Ministério da Saúde num processo que envolve pactuação local, regional e estadual e a participação do controle social.

A segunda fase consiste na etapa de desenvolvimento do conjunto de ações que serão empreendidas pelas Equipes de Atenção Básica, pelas gestões municipais e estaduais e pelo Ministério da Saúde, com o intuito de promover os movimentos de mudança da gestão, do cuidado e da gestão do cuidado que produzirão a melhoria do acesso e da qualidade da Atenção Básica. Esta fase está organizada em quatro dimensões (Auto avaliação; Monitoramento; Educação Permanente; e Apoio Institucional).

A terceira fase consiste na avaliação externa que será a fase em que se realizará um conjunto de ações que averiguará as condições de acesso e de qualidade da totalidade de municípios e Equipes da Atenção Básica participantes do Programa.

E, finalmente, a quarta fase é constituída por um processo de pactuação singular das equipes e dos municípios com o incremento de novos padrões e indicadores de qualidade, estimulando a institucionalização de um processo cíclico e sistemático a partir dos resultados alcançados pelos participantes do PMAQ.

\section{Considerações Finais}

Avaliar o Sistema Único de Saúde em toda a sua complexidade é uma tarefa que requer olhar em várias escalas diferenciadas (municipal, regional, estadual e nacional) e implica, por conseguinte, a apreensão em pelo menos três dimensões distintas: avaliação da satisfação dos usuários e trabalhadores; dos serviços de saúde e a partir dos dados secundários estruturados a partir dos bancos nacionais do SUS.

As estratégias aqui apresentadas, IDSUS, PNASS, PMAQ e as pesquisas de satisfação do usuário em curso no Ministério da Saúde buscam dar sinergia a um complexo sistema de avaliação que tenta abarcar as principais matizes do SUS.

Por outro lado, há um consenso entre os vários experts que, ao longo de décadas vem construindo o processo de avaliação do SUS Brasileiro, que a informação e a vigilância no campo da saúde são elementos-chave para a qualificação do fazer em saúde. Pensar em um Sistema de Avaliação só ganha relevância se esse processo estiver vinculado à melhoria da capacidade de gestão dos vários componentes do Sistema Único de Saúde brasileiro. A expectativa desta política é que estejamos avançando nesse caminho; a meta é estimular avanços ainda maiores nessa direção. 